\title{
Machine learning and registration for automatic seed localization in 3D US images for prostate brachytherapy
}

\author{
Hatem YOUNES ${ }^{1}$, Jocelyne TROCCAZ ${ }^{1}$ and Sandrine VOROS ${ }^{1,2}$ \\ ${ }^{1}$ Univ. Grenoble Alpes, CNRS, TIMC-IMAG, F-38000 Grenoble, France \\ ${ }^{2}$ Grenoble INP,INSERM, F-38000 Grenoble, France
}

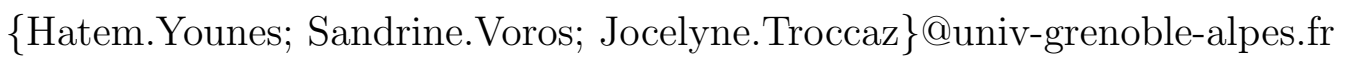

\begin{abstract}
Purpose: New radiation therapy protocols, in particular adaptive, focal or boost brachytherapy treatments, require determining precisely the position and orientation of the implanted radioactive seeds from real-time ultrasound (US) images. This is necessary to compare them to the planned one and to adjust automatically the dosimetric plan accordingly for next seeds implantations. The image modality, the small size of the seeds and the artifacts they produce make it a very challenging problem. The objective of the presented work is to set-up and to evaluate a robust and automatic method for seed localization in 3D US images.

Methods: The presented method is based on a pre-localization of the needles through which the seeds are injected in the prostate. This pre-localization allows focusing the search on a region of interest (ROI) around the needle tip. Seeds localization starts by binarizing the ROI and removing false positives using respectively a Bayesian classifier and a Support Vector Machine (SVM). This is followed by a registration stage using first an Iterative Closest Point (ICP) for localizing the connected set of seeds (named strand) inserted through a needle, and secondly refining each seed position using Sum of Squared Differences (SSD) as a similarity criterion. ICP registers a geometric model of the strand to the candidate voxels whilst SSD compares an appearance model of a single seed to a subset of the image. The method was evaluated both for 3D images of an Agar-agar phantom and a dataset of clinical 3D images. It was tested on stranded and on loose seeds.

Results: Results on phantom and clinical images were compared with a manual localization giving mean errors of $1.09 \pm 0.61 \mathrm{~mm}$ on phantom image and $1.44 \pm 0.45 \mathrm{~mm}$ on clinical images. On clinical images, the mean errors of individual seeds orientation was $4.33 \pm 8.51^{\circ}$.
\end{abstract}

Conclusions: The proposed algorithm for radioactive seed localization is robust, tested on different US images, accurate, giving small mean error values, and returns the 5 cylindrical seeds degrees of freedom.

Keywords: Prostate brachytherapy, Radioactive seed localization , 3D Ultrasound image, Bayesian classifier, support vector machine (SVM), iterative closest point (ICP), Sum of squared Differences (SSD) 
$\begin{array}{lr}\text { I. Introduction } & 1\end{array}$

I.A. State of the art . . . . . . . . . . . . . . . . . . . 2

II. Materials and Methods $\quad 5$

II.A. Needle detection . . . . . . . . . . . . . . . . . . . . . . 5

II.B. Seeds localization . . . . . . . . . . . . . . . . . . . 6

II.B.1. Thresholding: Binary Bayesian classifier . . . . . . . . . . . 6

II.B.2. False positive voxels removal: Support Vector Machine (SVM) . . . . 7

II.B.3. Global stranded seeds localization . . . . . . . . . . . . . . . . . 9

II.B.4. Improvement of individual seeds localization . . . . . . . . . . . . . . 9

II.C. Materials ........................ . . 10

II.C.1. Phantom experiments . . . . . . . . . . . . . . . . 10

II.C.2. Clinical experiments . . . . . . . . . . . . . . . . 11

$\begin{array}{ll}\text { III. Results } & 11\end{array}$

III.A. Phantom image . . . . . . . . . . . . . . . . . . . . . . . . 11

III.A.1. Global strand localization ... . . . . . . . . . . . . 11

III.A.2. Pose refinement . . . . . . . . . . . . . . . . . . . . . 12

III.B. Clinical images . . . . . . . . . . . . . . . . . . 13

$\begin{array}{ll}\text { IV. Discussion } & 13\end{array}$

$\begin{array}{lr}\text { V. Conclusion } & 16\end{array}$

$\begin{array}{lr}\text { VI. Acknowledgments } & 16\end{array}$

$\begin{array}{ll}\text { References } & 17\end{array}$

$\begin{array}{lr}\text { VII.Figures } & 23\end{array}$ 
63

\section{Introduction}

Prostate cancer is the $3^{\text {rd }}$ most frequently diagnosed cancer in men worldwide, accounting

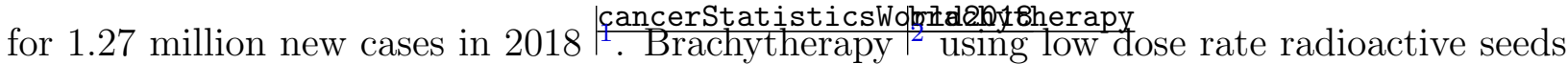
is an effective treatment for low risk prostate cancer. It aims at delivering a specific and homogeneous radiation dose to the prostate whilst limiting the dose delivered to organs at risk (urethra, rectum and bladder). This requires a planning step determining the number and optimal positions of seeds from pre-operative images: most often transrectal US images. In a typical brachytherapy procedure ${ }^{1}$ parallel needles are inserted into the patient's prostate through the skin of the perineum with the help of a guiding grid named template. Once the needle tip reaches the wanted position, the seeds are released through the needle. Two strategies are possible depending on the type of seeds: stranded seeds are pre-assembled with spacers using connectors and released as a whole for each insertion direction. On the opposite, loose seeds are released one by one, which makes needle retraction more complex and progressive. Needle insertion and seed implantation are performed under the control of transrectal US imaging (generally reconstructed in 3D from translated axial 2D US).

In practice, seed implantation is performed manually with some imprecision which results from operator variable expertise, prostate motion and deformation, edema, needle deflection, etc. Stranded seeds reduce delivery inaccuracy and seed migration compared to loose seeds. However, in both cases, the brachytherapy procedure may require a planning update, at some stage, taking into account the real position of already implanted seeds. Detecting them most often requires human intervention. In this paper we propose a novel method for automatic seeds localization in 3D US images. Connected to a fast replanning $\frac{\text { in }}{3}$, this allows to envision a fully automatic adaptive brachytherapy, that is an intra-operative update of the seeds placement planning, based on the localization and actual dosimetry map of the previously implanted seeds.

Detecting the seeds is a challenging task due to their small volume (cylinder: $\varnothing=0.8$ $\mathrm{mm}$ and $5 \mathrm{~mm}$ in length for $\mathrm{I}^{125}$ seeds) and to the low quality of the US imaging modality.

\footnotetext{
${ }^{1}$ https://www .mayoclinic .org/tests-procedures/prostate-brachytherapy/multimedia/ permanent-prostate-brachytherapy/img-20008710
} 


\section{I.A. State of the art}

The accurate localization of seeds is an active field of research. Several approaches have been proposed operating on various image modalities, like magnetic resonance (MRI), X-ray images (computed tomography CT or fluoroscopy) and ultrasound images (US). Because of its excellent soft-tissue contrast, MRI is essentially used for diagnostic. It could be also used for seed localization post-operatively: Kuo et al. ${ }^{\frac{M R I}{d}}$ eveloped an algorithm based on the blob detection technique using the Laplacian of a Gaussian (LoG). Generally the CT images are used to perform post-implant dosimetry one month after seeds implantation. In this context, N'Guyen et al. CTseedSegmentation images using K-means and principal component analysis (PCA) techniques; the method allows to separate seeds grouped in clusters, a situation that may occur with loose seeds. MRI only and CT-MRI fusion-based works are proposed to localize seeds post-operatively using Deep Neural Network (DNN) methods for low-dose-rate prostate brachytherapy Whilst CT and MRI may be exploited for pre-operative diagnostic or planning or postoperative assessment, they cannot be used for intra-operative control of seed position. A possible intra-operative solution is to obtain the real position from X-ray images acquired

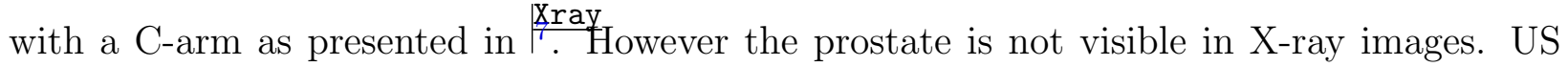
imaging is thus the effective modality used today to guide brachytherapy as it acquires realtimes images, which is particularly relevant in the context of moving and deformable soft tissue.

In this section we describe some of the few methods proposed in the literature to localize seeds in US images. Because seeds are injected through needles, some seed localization methods start with the needle tip localization. This allows building a ROI to be explored.

Wei et al. SeedLocalization1 1 proposed a workflow composed of 6 iterative instructions. First steps aim to detect the needle, build the ROI and threshold the obtained volume. Then, neighboring candidate voxels, potentially corresponding to seeds, are grouped into clusters. Seeds are localized by detecting the center and the orientation of each cluster using the PCA method. Finally, clusters which dimensions do not correspond to seed dimensions are removed. These steps are repeated until all implanted seeds have been localized. The method was tested on Agar-agar and chicken phantoms with a CT-based ground truth. Such a method may only be used to localize loose seeds since strands dimensions vary with their composition. 
As pointed by the authors, selecting the threshold may be very challenging since US images vary a lot from patient to patient.

In a rather similar way, Wen et al SeedLocalizationNeedle2

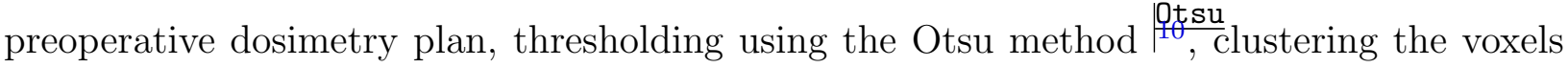
and pruning the set of clusters using dimension information. Needle track based on Hough Transform (HT) is used a posteriori to filter the seed detection results. The method was evaluated on a commercial tissue equivalent phantom. However, the information deduced from the preoperative dosimetry plan may be inaccurate to construct the ROI. Indeed, as the inserted needle may deviate from the target, seeds may be released away from the planned positions. The challenges of real clinical images are also underlined by the authors.

Based on a DNN, Holupka et al. PNN $^{\mathbb{N}}$ determine the position of the seeds from two dimensional transaxial transrectal US clinical images. A common DNN, called DetectNet was trained using 950 US images and tested on 90 validation US images. The network was trained from a data base of 2D clinical images manually segmented. The results on 10 patients were compared with the corresponding positions in CT images to obtain an average error of $2.29 \mathrm{~mm}$. However, CT images were acquired one to three months after the day of the implant where prostate gland may inflate causing seeds positions displacement. To compensate this, obtained seeds positions were transformed according to an empirical scaling factor representing the prostate shape change.

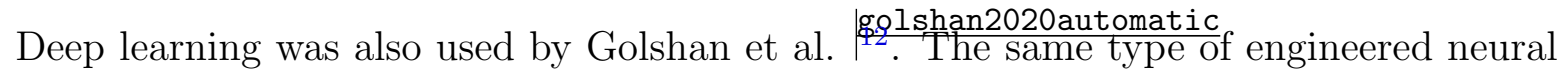
network $(\mathrm{CNN})$ was applied to locate the needle and its implanted stranded seeds. The learning phase is based on rather small number of 3D images of anonymous patients: 13 images containing 238 seeds. It was carried out with patches, which are 3D cubic subregions of the ultrasound images, representing models for a 'seed' region and of a 'non seed' region. Localization results were compared with an expert manual localization on ultrasound images giving $F 1 \_s c o r e=0,7$ (seed considered as true positive when its localization error is smaller than $2.5 \mathrm{~mm})$.

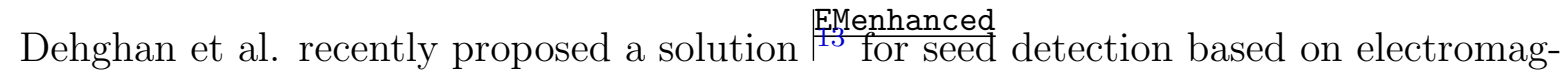
netic (EM) needle tracking, which allowed recording of seed dropping locations. A 3D US volume was obtained after each injection. Then, successive registered volumes were subtracted to obtain a difference volume. There, seed candidates were detected using their 
extended reverberations as a signature. Finally, false positive seeds were removed by comparing their locations with the expected location which corresponds to the seed dropping locations. However, the transfer to clinical routine of EM tracking (and associated needle modification) is not straightforward.

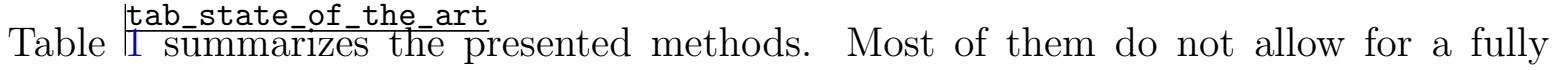
automatic binarization. Methods also differ regarding the number of localization parameters that are determined. Ideally, the complete pose of a cylindrical seed requires 5 parameters $\left(x, y, z, \theta_{x}, \theta_{y}\right)$. Given the cylindrical shape of the seed, $\theta_{z}$ is meaningless and an arbitrary value can be chosen. Many methods consider only the position of the seed (3 parameters) and this may be sufficient when dose calculation relies on very simple $1 \mathrm{D}$ model (the seed is considered as a point source and the delivered dose is spherical). However, since very long

rivard2004update dose models for cylindrical seeds. Taking full benefit of such models requires knowing the orientation of the seed. Collins Fekete et al. Scollins2014quantifying errors may have in impact on the dose delivered to organs at risks. Moreover adaptive brachytherapy involves more complex dose models $\frac{\text { inversePlanning }}{3}$ requiring a full localization of the seed. Finally, published methods differ in their level of evaluation. Whilst, all the methods cited in tab state of the art table 1 compare to CT based ground truth, most of them are limited to phantom experiments. Only the deep learning-based methods present results on real patient images.

In this paper, we aim at determining the 5 pose parameters of stranded seeds. An initial stage consists in localizing the needle before seed insertion to reduce the ROI to the relevant region (see $\S \|$ il.A.). In order to adapt to local variations in the image and to the patient, we propose an automatic thresholding method based on Bayesian classification (see $\S$ 无.cc:Bayesi.1.). False positive voxels, that may be selected by thresholding, then detected and removed using

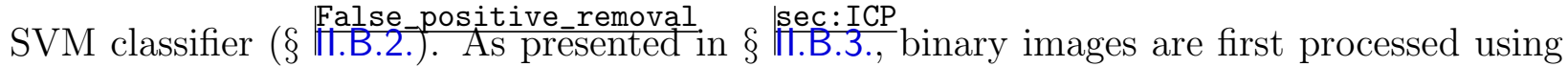
planning information to localize stranded seeds in the ROI as a whole. In a second stage explained in section IIt.B.4. orientations individual seeds are precisely localized by locally comparing the grey level of the original image to a seed 3D US image template. The approach was evaluated on two experimental setups: a phantom experiment allowed us to compare our localization method of dummy seeds in an Agar-agar phantom to a a reference manual segmentation sec:phantom_experiments $(\S$ il.C.1.). The method was also tested on routine clinical images and also compared to a reference manual segmentation ( $\S$ II.C.C.2.). The associated results are presented in section 
sec: Phantosed mazinireshltwsages

208

\section{Materials and Methods}

Seeds localization begins with a needle detection which is an essential step to build the ROI where seeds are released. First, we introduce the employed method to localize a needle in 3D US images which we previously developed. Then we explain the seed localization method modeled by the workflow of Fig. Ifig:

\section{II.A. Needle detection}

Needle detection is done using the previous work ${ }^{\text {purWhork }}$ based on binary Bayesian classification. We proposed a method to localize needle in 3D US images. Needle voxels within US volumes are brighter than the other voxels, and form a voxel class selected according to the Bayesian classifier:

$$
\pi_{X_{n}} P\left(I(x)=y \mid X_{n}\right) \underset{X_{b}}{\stackrel{X_{n}}{\gtrless}} \pi_{X_{b}} P\left(I(x)=y \mid X_{b}\right)
$$

- $\quad X_{n}=$ needle voxel class.

- $\quad X_{b}=$ background (non-needle) voxel class.

- $y=I(x)=$ voxel intensity $x$.

- $\pi_{X_{i}}=$ prior probabilities.

- $P\left(I(x)=y \mid X_{i}\right)=$ Gaussian probabilities.

Where: a voxel $x$ is classified in $X_{n}$ if the posterior probability of $X_{n}$ is larger than that of $X_{b}$. The way parameters of the probabilistic model are estimated and a curve representing the needle is fitted to data is fully explained in purWork . The proposed method was successfully tested on anonymized 3D US clinical images. Fig. 2 shows a typical result example. In order to obtain a good estimate of the dropping location of seeds, with respect to the previous work ${ }^{\text {ourWork }}$, we added a new step to refine the needle tip search along the detected curve. The tip is the last voxel along the curve where

$$
P\left(X_{n} \mid I(x)\right) \leq P\left(X_{b} \mid I(x)\right)
$$




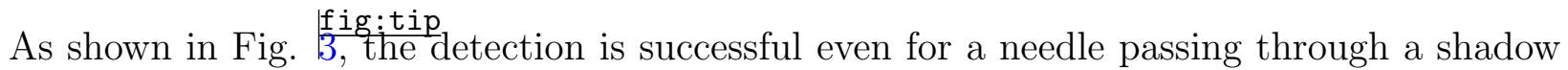
region, which is a dark region that corresponds to some reflected signals attenuation. Needle detection allows to build the ROI where seeds are supposed to be located. As shown in Fig. 4 fig: References released strand supposed to be inside. As explained in $\S$ III.B. ICP. and shown in table 2 , strab_strands compositions and lengths are recoverable from the treatment planning. The next subsection describes the method for seeds localization in the ROI.

\section{II.B. Seeds localization}

The current proposed method has been designed for stranded seeds localization. The work-

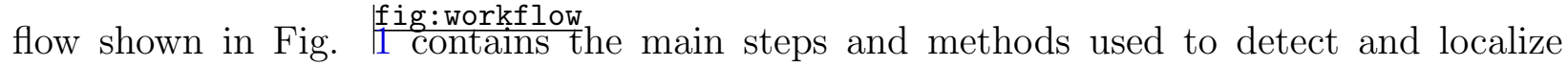
oriented seeds. We detail each step separately in the following subsections. The first typical step for object detection is to separate it from the background.

\section{II.B.1. Thresholding: Binary Bayesian classifier}

A thresholding step aims to separate seeds voxels from background. The classical method consists in defining a threshold value $\tau$ and classifying as follows:

$$
\text { Seeds }=\{x: I(x) \geq \tau\}
$$

$$
\text { Background }=\{x: I(x)<\tau\}
$$

Because of the presence of high intensity artifacts and the high variability of grey levels in US images, defining thresholds for binarization is very challenging. This is why we propose to use a Bayesian classifier to perform binarization without a static threshold definition. In a way similar to needle detection, seeds and background voxels are described through two separated peaks which can be modeled using an additive Gaussian Mixture Model (GMM).

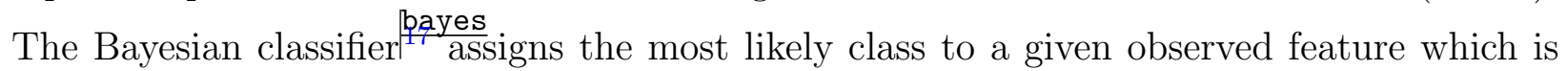
the voxel intensity.

$$
C_{\text {Bayes }}(y)=\underset{X_{i}}{\operatorname{argmax}} \quad P\left(C l=X_{i} \mid I(x)=y\right)
$$


Where $C l=\left\{X_{s}(\right.$ Seeds $), X_{b}($ Background $\left.)\right\}$, and $I(x)=y$ is the voxel intensity. According to Bayes theorem:

$$
P\left(C l=X_{i} \mid I(x)=y\right)=\frac{\pi_{X_{i}} P\left(I(x)=y \mid C l=X_{i}\right)}{P(I(x)=y)}
$$

Where $\pi_{X_{i}}$ are the prior probabilities and $P\left(I(x)=y \mid X_{i}\right)$ are the Gaussian probabilities. The intensities $I(x)$ are independent within each class, which justifies the Bayes theorem application. According to equations (3) and (4), nayes 2 alve Bayesian classifier assigns to each voxel the class which maximizes $\operatorname{argmax}\left\{\frac{\pi_{X_{s}} P\left(I(x)=y \mid C l=X_{s}\right)}{P(I(x)=y)}, \frac{\pi_{X_{b}} \quad P\left(I(x)=y \mid C l=X_{b}\right)}{P(I(x)=y)}\right\}$. Finally, binary Bayesian classification to be used is summarized as inequalities (

$$
\pi_{X_{s}} P\left(I(x)=y \mid X_{s}\right) \underset{X_{b}}{\stackrel{X_{s}}{\gtrless}} \pi_{X_{b}} P\left(I(x)=y \mid X_{b}\right)
$$

ineq:Bayes 1

Several parameters are required: $\left\{\pi_{X_{i}}, \mu_{i}, \sigma_{i}\right\}$ where $i=\{s, b\}$ and $\mu, \sigma$ are the Gaussian parameters. These parameters vary from one stranded seed to another even in the same image. According to a criterion of maximum likelihood, parameters are predicted by approaching as much as possible the distribution of seeds volume histogram. This is done via with the well known Expectation-Maximization algorithm (EM algorithm ${ }^{\text {EM }}$ ). EM is applied on a Gaussian mixture model to estimate the unknown parameters by an iterative process, which computes the maximum-likelihood of a given vector of features for each iteration. EM algorithm iterates parameters calculation until their convergence. Fig. fig:firștTwoSteps example.

\section{II.B.2. False positive voxels removal: Support Vector Machine (SVM)}

It naturally happens that some background voxels are assigned as seed voxels. In order to remove these false positives, we propose to apply an additional discriminator: Support Vector Machine (SVM), a learning classifier. In the case of binary classification, a SVM maximizes the margin between classes $C^{1}$ of false positive voxels and $C^{2}$ of true positive voxels $\left(C^{1} \cup C^{2}=X_{s}\right)$. It aims to find the hyperplane that separates classes optimally. In the linear case, the main equation to establish the hyperplane equation is:

$$
h(x)=w^{T} x+w_{0}
$$

where $x=\left(x_{1}, \ldots, x_{N}\right)$ is the input vector which contains voxel features, $w$ is the normal vector to the hyperplane and $w_{0}$ is the offset of the hyperplane from the origin along the 
normal vector $w$. Finally the last parameters $\left(w^{T}\right.$ and $\left.w_{0}\right)$ determine the classifier as follows:

$$
x \mapsto \operatorname{sign}\left(w^{T} \cdot x+w_{0}\right)
$$

The input vector $x$ is assigned to the appropriate class regarding to the sign of $\left(w^{T} \cdot x+w_{0}\right)$. It represents the position of $x$ with respect to the hyperplane. As mentioned previously, the input vector $x$ represents the input voxel to be classified. It contains the voxel features. Classification results depend on the selected features. Based on the specificity of brachytherapy application, we propose to use two gradient-based features: indeed, stranded seeds injection in the prostate follows the $\vec{Z}$ axis of the 3D US image, so the intensity change of seeds voxels according to $\vec{X}$ and $\vec{Y}$ could have high value, contrarily to others outliers voxels (Fig. f). fig: first The gradient magnitude $\left(\left|G_{x}\right|,\left|G_{y}\right|\right)$ along $\vec{X}$ and $\vec{Y}$ provide a good discriminative representation as shown in Fig. $\frac{\mid \text { fig: hyperplane }}{6}$ where $x=\left(x_{1}, x_{2}\right)=\left(G_{x}, G_{y}\right),\left\{G_{x}, G_{y}\right\}$ are the gradient magnitudes. The gradient magnitudes are computed from the original US image. The Sequential Minimal Optimization (SMO ${ }^{\text {SMO }}$ ) algorithm is used to compute the hyperplane equation parameters $\left(w^{T}, w_{0}\right)$. It transforms the problem of parameters prediction into the dual problem:

$$
h(x)=w^{T} x+w_{0}=\sum_{i=1}^{N} \alpha_{i} y^{(i)}<x^{(i)}, x>+w_{0}
$$

where

- $\left(x^{(i)}, y^{(i)}\right)=$ training data.

- $y^{(i)}=\left\{-1\right.$ if $x^{(i)} \in C^{1}, 1$ if $\left.x^{(i)} \in C^{2}\right\}$.

- $\alpha \in \Re^{N}=$ Lagrange multipliers.

- $\quad N=$ the training data length.

For training the SVM, we chose three ROI containing different stranded seeds compositions and different artifacts locations for which we calculated and labelled the features $\left(\left|G_{x}\right|,\left|G_{y}\right|\right)$. SMO was trained on the fusion of these three datasets to return the best separative hyperplane which maximizes the distance at the nearest data point of each group. A typical SVM discrimination result is shown in Fig. fig. 


\section{II.B.3. Global stranded seeds localization}

At this stage, strand voxels have been computed using a succession of two classifiers. As

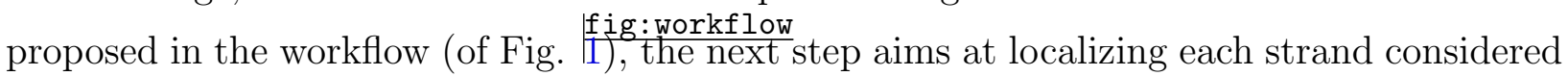
as a whole within selected voxels. This is done by rigidly registering a geometric model of the stranded seeds to the seed voxels detected in the previous stets, represented by their centers. This is performed using the Iterative Closest Point (ICP) algorithm ${ }^{\text {ICP }}$. One objective is to transform the 3D voxels already selected into a set of connected cylinders. A second very important objective is to distinguish seeds from spacers connecting them since their US appearance is very similar (Fig. 5 fig:firstTwosteps model of the strand injected through each needle. This model, which is a set of 3D surface points, describes the succession of cylinders that represent radioactive seeds linked through

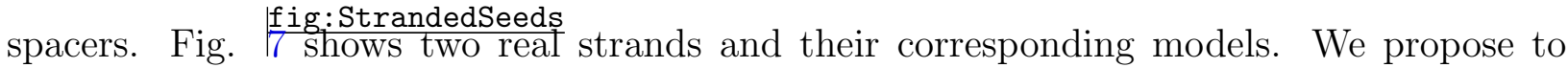
employ ICP to superimpose the two sets of 3D points. ICP requires a suitable initialization of the relative position of data to be rigidly registered. Since the closest known position of the strand to be localized is the needle tip, the model is initialized at needle tip coordinates (obtained as specified in III.A.) and is aligned with the insertion direction. It allows to find the optimal transformation representing the location of the strand as a whole:

$T_{\text {Strand }}=\left\{\theta_{x}^{\text {Strand }}, \theta_{y}^{\text {Strand }}, t_{x}^{\text {Strand }}, t_{y}^{\text {Strand }}, t_{z}^{\text {Strand }}\right\}, \quad$ where $\quad\left\{\theta_{x}^{\text {Strand }}, \theta_{y}^{\text {Strand }}\right\}$ and $\left\{t_{x}^{\text {Strand }}, t_{y}^{\text {Strand }}, t_{z}^{\text {Strand }}\right\}$ are respectively the rotations and the translations applied on the strand relatively to the ROI frame $\{\vec{X}, \vec{Y}, \vec{Z}\}$ (Fig. fig: Geferences the strands, it is impossible to determine the rotation around its $\vec{Z}$ axis. This information is meaningless and an arbitrary value can be given.

\section{II.B.4. Improvement of individual seeds localization}

After the ICP convergence, the optimal position of the strand model with respect to the binary image information is obtained, where all seeds are well aligned. However, in practice, stranded seeds may not remain straight, as the linkers may break during the insertion. It may result in subsets of linked seeds with different orientations as shown in Fig. $\frac{\mid f i g ; \text { firstTwoSteps }}{5 . A s ~ s e e d s}$ mislocations may modify the delivered dose, an additional stage is necessary and consists in refining each seed pose separately. From that stage, spacers are no longer considered. Knowing precisely the pose of each seed would allow to update the dosimetry and replan, 
when necessary, the positions of the next seeds to be injected. This refinement is an imagebased rigid registration comparing an appearance model of a seed $V_{m}$ to a subset $V_{s}$ of the real gray level image. $V_{s}$ is computed from the location of the strand obtained in $\stackrel{\text { sit. }: \text { : ICP }}{\text { I.. }} V_{m}$ was obtained by selecting a clearly visible seed in an image among those of our dataset (Fig. f ig: VS 8). This appearance model was applied for all localized seeds.

$$
T_{\text {seed }}=\underset{T}{\operatorname{argmin}} \sum_{x, y, z}\left(V_{m}(x, y, z)-T\left(V_{s}(x, y, z)\right)\right)^{2}
$$

The optimal transformation $T_{\text {seed }}$ is the one that minimizes the sum of squared differences (SSD) value between the model volume $V_{m}$ and the seed volume $V_{s}$ moved according to $T$ where $T=\left\{\theta_{x}^{\text {Seed }}, \theta_{y}^{\text {Seed }}, t_{x}^{\text {Seed }}, t_{y}^{\text {Seed }}\right\}$. Similarly to the strand, $\theta_{z}^{\text {Seed }}$ cannot be determined and is meaningless. Moreover, because seeds, connectors and spacers have similar response and

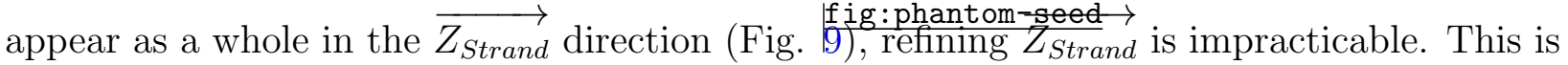
why neither $t_{z}^{\text {Seed }}$ nor $\theta_{z}^{\text {Seed }}$ are considered at this stage. $\theta_{z}^{\text {Seed }}$ can be arbitrarily chosen and $t_{z}^{\text {Seed }}$ value is kept similar to what was computed in the previous stage.

\section{II.C. Materials}

The approach was tested both on phantom image and on clinical images. This section describes the data acquisition and validation protocol for both types of experiments.

\section{II.C.1. Phantom experiments}

Using a proportion of $2.8 \%$ of Agar-agar contained in a plastic box (Fig. Ifig), wOELIS_Trinity a phantom sufficiently rigid to avoid any movement of released seeds. We created a preoperative dosimetry plan where 8 needles were inserted. Each of them was used to release one strand. The strands composition is given in table 2 . The phantom with the implanted seeds was scanned using the 3D side-fire ultrasound probe of the TRINITY ${ }^{\circledR}$ (Koelis, Meylan, France) assistance workstation for prostate interventions shown in Fig. 1 I0. 3D 10 images were produced using the probe internal motor moving a linear array transducer on $170^{\circ}$ around the probe axis. The center frequency of the transducer was set to $7 \mathrm{MHz}$, and the capturing depth was set to $80 \mathrm{~mm}$. The size of generated images was $95 \times 95 \times 80 \mathrm{~mm}$, with a voxel size of $0.3125 \times 0.3125 \times 0.3125 \mathrm{~mm}$. Seeds in the phantom image are as shown in Fig. $\frac{\text { fig: } \text { : phantom-seed }}{\text {. }}$ 
To measure the error of the presented seed localization method, we compared automatic localization with a manual segmentation considered as a ground truth. We evaluated the precision of the manual segmentation by repeating it at 7 distant times, and obtained a standard deviation of $0.66 \mathrm{~mm}$.

\section{II.C.2. Clinical experiments}

The algorithm was also tested on anonymized 3D US images of two patients. Each patient's prostate with the implanted seeds was scanned using a biplane endocavity Z848 TRUS probe within the Medical Flex Focus 500 ultrasound workstation. Its biplane transducer acquired 75 axial images of resolution $600 \times 580$ and a 3D image was reconstructed with a voxel size of $0.26 \mathrm{x} 0.26 \mathrm{x} 1 \mathrm{~mm}$. The evaluation was also done by comparing the automatic localization with a manual segmentation considered as a ground truth. Because of the very low quality of the images (Fig. f fig:firstTwoSteps be reliably done. 4 stranded seeds were selected from the data of patient 1 containing in total 11 seeds. For the image of patient 2, 3 stranded seeds containing 6 seeds were processed. Detailed compositions of stranded seeds are presented in table th. We have first measured the variability of the manual segmentation by repeatedly segmenting the centers of three seeds in the two images, at 7 distant times. The obtained standard deviation was $0.7 \mathrm{~mm}$.

\section{Results}

After describing the results of our method using the phantom validation protocol, we present the corresponding results on 3D US clinical images. The parameters values used for each US image type are summarized in table $\frac{\operatorname{tab}}{4 .}$

\section{III.A. Phantom image}

\section{III.A.1. Global strand localization}

The errors on the seed localization are computed as the Euclidean distances between the centers of the automatically detected and manual identified seeds. Fig. $\frac{\text { fig: boxplotPhantom }}{12}$ presents the statistics of these errors before $(3.08 \pm 1.55 \mathrm{~mm})$ and after $(1.55 \pm 0.80 \mathrm{~mm})$ global strands 
localization where the unknowns are $\left(t_{x}^{\text {Strand }}, t_{y}^{\text {Strand }}, t_{z}^{\text {Strand }}, \theta_{x}^{\text {Strand }}, \theta_{y}^{\text {Strand }}\right)$. The errors before global strands localization correspond to the distance between the seeds of the strand model initialized at the needle tip and the ground-truth positions.

\section{III.A.2. Pose refinement}

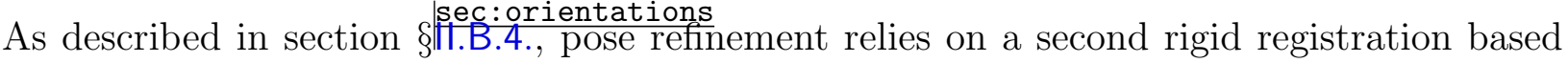
this time on voxel intensity differences. Fig. $\frac{f i g \text { : } 12 \text { givesplotPhantom }}{12}$ the corresponding boxplot before and after seed pose refinement where the unknowns are $\left(t_{x}^{\text {Seed }}, t_{y}^{\text {Seed }}\right)$ (more details in figure S1). The global error is measured as the Euclidean distance between the seeds centers. The mean error is $1.09 \pm 0.61 \mathrm{~mm}$. It was not possible to refine the orientations of individual seeds implanted in the phantom because strands did not break, and the strand remained perfectly straight. Therefore, we tested pose refinement on phantom data only for $t_{x}^{\text {Strand, }}$ $t_{y}^{\text {Strand }}$. We simulated orientation errors by rotating the seed volume $V_{s}$ and studied how well the transformation parameters could be recovered. Seeds of a strand do not deviate a lot compared to loose seeds. Their deviations are mainly between $-20^{\circ}$ and $+20^{\circ}$ relative to a direction according to an orientation effects study on the post-planning dosimetry of low-dose-rate prostate brachytherapy ${ }^{\mid c o l l i n s 2014 q u a n t i f y i n g}$. Therefore tests were performed between $-20^{\circ}$ and $+20^{\circ}$. Based on the template grid dimensions where needle holes are located every $5 \mathrm{~mm}$ in $\vec{X}$ and $\vec{Y}$, a natural dimension in $\vec{X}$ and $\vec{Y}$ of $V_{s / m}$ would not exceed $\sim 2 \times 5 \mathrm{~mm}$ to ensure that $V_{s / m}$ contain only one seed. The only constraint along $\vec{Z}$ is the seed length. Taking into account the linker, we chose $6 \mathrm{~mm}$ as the $3^{r d}$ dimension of $V_{s / m}$. Given the voxels resolution, we ended with $V_{s / m}$ dimensions of $11.25 \times 10.31 \times 9 \mathrm{~mm}$. The seed appearance within $V_{m}$ is shown in Fig. Ifig: orientations

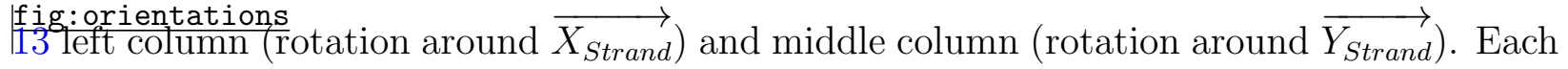
row corresponds to a seed where the red curve contains the applied angles and the blue curve

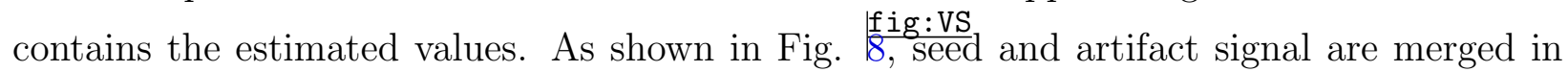
the transversal plane, contrarily to the sagittal one where the seed is clearly visible. As a rotation around $\overrightarrow{Y_{\text {Strand }}}$ corresponds to a motion in the transversal plane, this rotation angle cannot be accurately refined by our method because of the artifacts, as illustrated by Fig. fig:orientations 13, middle column. Although the information exists, the estimation of the seed orientations around $\overrightarrow{Y_{\text {Strand }}}$ failed with the chosen dimensions of $V_{s / m}$ : indeed those dimensions affect the orientations estimation, when they are of the same order of magnitude as the artifacts 
dimensions. This was confirmed by extending the dimensions of $V_{s}$ and $V_{m}$ to $18.75 \times 10.31 \times 9$ $\mathrm{mm}(60 \times 33 \times 29$ voxels $)$ instead of 11.25x10.31x6 mm (36x33x19 voxels) and running the same experiments. The simulation results for the rotation around $\overrightarrow{Y_{\text {Strand }}}$ with this new $V_{s / m}$ size are shown in Fig. Ifig:orientations and $2.44^{\circ}$. It can be concluded that the small cropping of $V_{s}$ and $V_{m}$ is problematic because (sec:discussion of the artifact extending laterally over the volumes. This will be discussed in section IV..

\section{III.B. Clinical images}

fig:boxplotpatients

Fig. 14 presents the localization errors before global strand localization $(2.36 \pm 1.11 \mathrm{~mm})$, before $(1.59 \pm 0.55 \mathrm{~mm})$ and after $(1.44 \pm 0.45 \mathrm{~mm})$ individual seeds refinement (more details in figure S-2). The unknown parameters are $\left(t_{x}^{\text {Seed }}, t_{y}^{\text {Seed }}, \theta_{x}^{\text {Seed }}\right)$. Table 3 contains the seeds orientations refinement compared with the manual estimation. As explained in \$III.A.2., it was impossible to refine the rotation around $\overrightarrow{Y_{\text {Strand }}}$ axis. Rotation could be taken into account to some extent if the US images were acquired during seed insertions, but here the US data was acquired post-operatively with all seeds implanted. We will discuss this point in sec:discussion this point in $\oint \|$..

\section{Discussion}

Challenges in seed localization from 3D US images are essentially due to the low quality of the imaging modality and the small seed volume. Thus, some echogenic structures can mimic seed appearance and some seeds brightness are affected when they are in a shadow region. In this paper we aimed to overcome these difficulties by introducing an automatic seed localization using different machine learning and rigid registration algorithms.

An accurate segmentation of candidate seeds voxels is mandatory for the localization of the seeds. To do so, we performed a thresholding using a Bayes classification, followed by a false-positives removal using an SVM. The EM algorithm of the Bayes classification converges to local optima, and therefore requires a careful initialization of the means and variances parameters. How this initialization is handled is fully described in purWork was used for seed localization to obtain the adequate initialization values summarized in table $\frac{\text { tab:parameters }}{4 .}$ The following SVM false-positives removal step is a refinement which could not 
replace the Bayes Classification step. Indeed, SVM would then be applied to the original gray-level voxels, resulting in a huge amount of input points. This would be computationally expensive (estimation of the gradients of all the non-zero voxels of the US image). Moreover, this would reduce the precision of the estimation, as it would increase the number of points close to the hyperplane.

Regarding seeds localization, it has been shown in $\S$ siti.A. pose . 2 . that the orientation around $\overrightarrow{Y_{\text {Strand }}}$ could not be refined by the image-based local registration because of the US artifacts and the small size of $V_{s}$ and $V_{m}$. An incremental procedure would be a potential solution for extending the volumes of $V_{s}$ and $V_{m}$. For the first released strands, it would be performed without overlaps. Then, taking into account the neighbor potential seeds already detected would allow to enlarge $V_{m}$. The incremental procedure could also allow to introduce priors on previously detected seeds to modify this last stage. Of course, this would need further developments and testing.

The presented method was evaluated using two different US acquisition devices, on two different types of data (phantom and clinical), resulting in quite different image contents and quality. Despite this variety, only a few of the different parameters used to establish the classification and registration models needed to be tuned: the initialization values for the parameters estimations of the Bayes classification using EM depend on the probe devices, as illustrated by table thab:parameters 4 . Neither the SMO initialization values (false-positives removal using an SVM) nor the ICP initialization values (for the global strand registration) had to be adapted. SMO was trained using phantom data, but the estimated hyperplanes for phantom or clinical images are very close, despite the differences in image content, thanks to our choice of features. The only other device-dependent element is the seed appearance model $V_{m}$. We selected for each image type the most generic appearance model. Adapting the few device dependent parameters (EM initialization values and $V_{m}$ model) to new US imaging devices would be quite straightforward although additional pre-clinical experiments would be needed.

Despite the different experimental conditions, such as the probe technologies which provide different US images types (2D or 3D), qualities and resolutions, we try to compare the current work with the state of the art. Clinical images are used only in the deep learning-

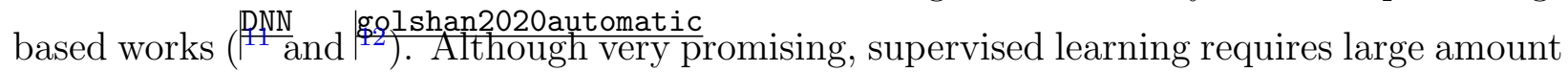


of precisely labeled clinical images to show good performance and ability to generalize: this may be a big issue as testify the published papers. The seed locations are determined by a commercial software in CT volume in ${ }^{\text {DNN }}$ and by an expert manual identification on US images in golshan2019automatic . Obtained results are within $2.29 \mathrm{~mm}$ and $2.5 \mathrm{~mm}$ (used to calculate F1_score)

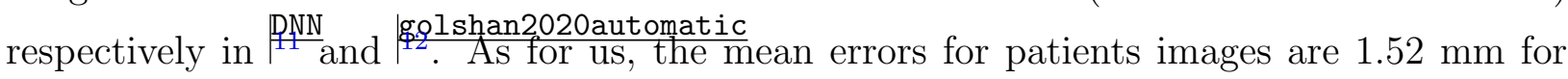
the $1^{\text {st }}$ patient and $1.36 \mathrm{~mm}$ for the $2^{\text {nd }}$ one. These good results need to be confirmed on larger clinical data. Concerning other mentioned methods, different types of phantom images are used (summarized in table 1 ). Seeds localization in phantom images is more accurate than that in a biological tissues images. For example, it is approximately $1.03 \mathrm{~mm}$ using Agar-agar phantom and $1.66 \mathrm{~mm}$ in chicken phantom SeedLocalization1

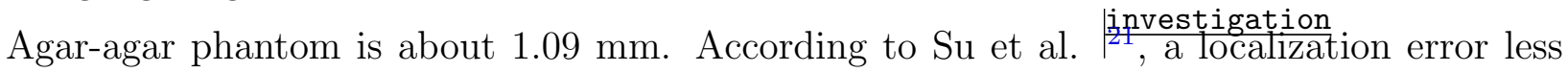
than $2 \mathrm{~mm}$ is customarily considered to be acceptable. They investigated the effects of a seed mislocation on the dosimetry accuracy and showed that an error of $2 \mathrm{~mm}$ accounts for less than $5 \%$ deviation in the dose delivered to $90 \%$ of the prostate volume. Collins Fekete et al. collins2014quantifying to organs at risks. Moreover, new focal treatments appear where a reduced number of seeds are inserted in an hemi-gland or even more locally for instance for a boost secondary treatment. Al-Qaisieh et al. 22015 dosimetry accurate localization of seeds for focal treatments. The presented method could contribute to such longer term applications.

As mentioned before, the use of stranded seeds decreases the implantation errors since it brings them together to make a single injection per needle. We tested the algorithm on various strand compositions. N8 of the table 2 released a strand composed just by one seed. Also, the composition of S1 of the table 3 is just one seed. It proves that this method, contrarily to the state of the art methods, could be a solution for both stranded and loose seeds localization. However if loose seeds are clustered the method would fail as the other methods presented in $\S$ It.A.. This question was addressed by N'Guyen et al. for CT images $\mid$ TseedSegmentation , and we could consider adapting their approach for the case of US images. Most

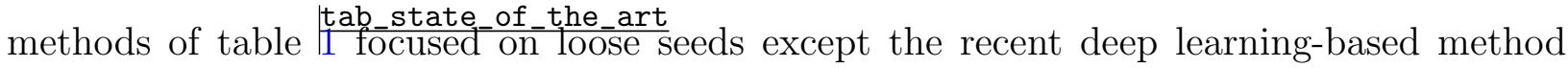
golshan2020automatic problems considering the inability to differentiate between spacers and seeds which have similar response and appearance in the US images. 
The proposed workflow has been specifically designed for stranded seeds localization in US images. It would probably not be directly applicable to other imaging modalities (MRI or CT) but several components of the approach could be generalized to another modality. Combining US information with other intra-operative data such as cone beam computed tomography (CBCT) image could be envisioned but the benefit/complexity ratio has to be carefully evaluated.

Regarding the clinical applicability of the presented method, several protocols can be envisioned. In a first stage, the automatic localization could simply happen when the peripheral seeds have been injected. This would allow re-planning for central seeds. However, ideally, the adaptive planning would be done much more often. In a way very similar to robot-assisted brachytherapy $\frac{\mid \text { cunna2010toward }}{4}$ where the robot generally handles a single needle and requires repeating needle insertion-seeds injection, re-planning could occur before each new needle insertion.

\section{Conclusion}

Adaptive brachytherapy requires an intra-operative update of the seeds placements, for which localizing the seeds is essential. We proposed an image-based solution for the localization (position and orientation estimation) of stranded seeds, which could also be applied to loose seeds. Our validation was performed both on phantom and clinical data, with two different US imaging devices. Our results are encouraging as the position accuracy is in-par with clinical requirements, and the orientation accuracy is improved compared to state of the art. Improvements and more extensive evaluations on clinical images will be needed to further validate the approach.

\section{Acknowledgments}

We thank LATIM and Brest University hospital for giving access to patient images. This was made possible through the clinical protocol FOCUS NCT03160365 "Innovative planning and guidance system for focal prostate brachytherapy. This work was partly supported by the French ANR within the Investissements dAvenir program (Labex CAMI ANR-11-LABX) 
522

and through the FOCUS (ANR-16-CE19-0011) and MIAI@Grenoble_Alpes (ANR-19-P3IA0003) projects.

\section{References}

1 F. Bray, J. Ferlay, I. Soerjomataram, R. L. Siegel, L. A. Torre, and A. Jemal, Global cancer statistics 2018: GLOBOCAN estimates of incidence and mortality worldwide for 36 cancers in 185 countries, CA: a cancer journal for clinicians 68, 394-424 (2018).

2 H. Raziee, A. Berlin, P. Chung, J. Helou, H. Jiang, J. M. Crook, and C. N. Catton, Permanent seed brachytherapy for low risk prostate cancer, long term outcome, and urinary toxicity, International Journal of Radiation Oncology Biology Physics 99, E262 (2017).

3 K. A. Mountris, D. Visvikis, and J. Bert, DVH-based inverse planning using Monte Carlo dosimetry for LDR prostate brachytherapy, International Journal of Radiation Oncology* Biology* Physics 103, 503-510 (2019).

4 N. Kuo, J. Lee, C. Tempany, M. Stuber, and J. Prince, MRI-based prostate brachytherapy seed localization, in 2010 IEEE International Symposium on Biomedical Imaging: From Nano to Macro, pages 1397-1400, IEEE, (2010).

5 H.-G. Nguyen, C. Fouard, and J. Troccaz, Segmentation, separation and pose estimation of prostate brachytherapy seeds in CT images, IEEE Transactions on Biomedical Engineering 62, 2012-2024 (2015).

6 R. Nosrati, M. Wronski, C. Tseng, H. Chung, A. Pejović-Milić, G. Morton, G. Stanisz, Postimplant Dosimetry of Permanent Prostate Brachytherapy: Comparison of MRI-Only and CT-MRI Fusion-Based Workflows, International Journal of Radiation Oncology* Biology* Physics 106, 206-215 (2019).

7 C. A. di San Filippo, G. Fichtinger, W. J. Morris, S. E. Salcudean, E. Dehghan, and P. Fallavollita, Intraoperative segmentation of iodine and palladium radioactive sources in C-arm images, International journal of computer assisted radiology and surgery $\mathbf{9}$, 769-776 (2014). 
8 Z. Wei, L. Gardi, D. B. Downey, and A. Fenster, Automated localization of implanted seeds in 3D TRUS images used for prostate brachytherapy, Medical physics 33, 24042417 (2006).

9 X. Wen, S. E. Salcudean, and P. D. Lawrence, Detection of brachytherapy seeds using 3D transrectal ultrasound, IEEE Transactions on Biomedical Engineering 57, 2467-2477 (2010).

10 N. Otsu, A threshold selection method from gray-level histograms, IEEE transactions on systems, man, and cybernetics 9, 62-66 (1979).

11 E. J. Holupka et al., The Detection of Implanted Radioactive Seeds On Ultrasound Images Using Convolution Neural Networks, (2018).

12 M. Golshan, D. Karimi, S. Mahdavi, J. Lobo, M. Peacock, S. Salcudean, I. Spadinger, Automatic detection of brachytherapy seeds in 3D ultrasound images using a convolutional neural network, Physics in Medicine \& Biology (2020).

13 E. Dehghan, S. Bharat, C. Kung, A. Bonillas, L. Beaulieu, J. Pouliot, and J. Kruecker, EM-enhanced US-based seed detection for prostate brachytherapy, Medical physics 45, $2357-2368$ (2018).

14 M. Rivard, B. Coursey, L. DeWerd, W. Hanson, M. Saiful Huq, G. Ibbott, M. Mitch, R. Nath, J. Williamson, Update of AAPM Task Group No. 43 Report: A revised AAPM protocol for brachytherapy dose calculations, Medical physics 31, 633-674 (2004).

15 C. Collins Fekete, M. Plamondon, A. Martin, É. Vigneault, F. Verhaegen, L. Beaulieu, Quantifying the effect of seed orientation in postplanning dosimetry of low-dose-rate prostate brachytherapy, Medical physics 41, (2014).

16 H. Younes, S. Voros, and J. Troccaz, Automatic needle localization in 3D ultrasound images for brachytherapy, in 2018 IEEE 15th International Symposium on Biomedical Imaging (ISBI 2018), pages 1203-1207, IEEE, (2018).

17 I. Rish et al., An empirical study of the naive Bayes classifier, in IJCAI 2001 workshop on empirical methods in artificial intelligence, volume 3, pages 41-46, (2001). 
ES4 18 A. P. Dempster, N. M. Laird, and D. B. Rubin, Maximum likelihood from incomplete 576 data via the EM algorithm, Journal of the Royal Statistical Society: Series B (Method577 ological) 39, 1-22 (1977).

SM9s 19 Z.-Q. Zeng, H.-B. Yu, H.-R. Xu, Y.-Q. Xie, and J. Gao, Fast training support vec579 tor machines using parallel sequential minimal optimization, in 2008 3rd international conference on intelligent system and knowledge engineering, volume 1, pages 997-1001, IEEE, (2008).

D. Chetverikov, D. Svirko, D. Stepanov, and P. Krsek, The trimmed iterative closest point algorithm, in Object recognition supported by user interaction for service robots, volume 3, pages 545-548, IEEE, (2002).

21 Y. Su, B. J. Davis, K. M. Furutani, M. G. Herman, and R. A. Robb, Dosimetry accuracy as a function of seed localization uncertainty in permanent prostate brachytherapy: increased seed number correlates with less variability in prostate dosimetry, Physics in Medicine and Biology 52, 3105 (2007).

22 B. Al-Qaisieh, J. Mason, P. Bownes, A. Henry, L. Dickinson, H. Ahmed, M. Emberton, S. Langley, Dosimetry modeling for focal low-dose-rate prostate brachytherapy, International Journal of Radiation Oncology* Biology* Physics 92, 787-793 (2015).

J A. Cunha, I C. Hsu, J. Pouliot, M. Roach III, K. Shinohara, J. Kurhanewicz, G. Reed, D. Stoianovici, Toward adaptive stereotactic robotic brachytherapy for prostate cancer: demonstration of an adaptive workflow incorporating inverse planning and an MR stealth robot, Minimally Invasive Therapy \& Allied Technologies 19, 189-202 (2010). 
Table 1: Comparative study about proposed methods for tab_state_of_the_art seeds localization.

\begin{tabular}{|c|c|c|c|c|}
\hline & Problem to solve & Methods & US images & Ground truth \\
\hline Wei et al. SeedLoca & $\begin{array}{l}\text { Loose seeds } \\
\text { lization1 } \\
\text { positions }+ \text { orientations } \\
\text { in 3D image }\end{array}$ & $\begin{array}{l}\text { Gray Level Change } \\
\text { GLC }>\mathrm{T}\end{array}$ & $\begin{array}{l}\text { Agar-agar phantom } \\
\text { Chicken phantom }\end{array}$ & CT images \\
\hline Wen et al. & $\begin{array}{l}\text { Loose seeds } \\
\text { alizationNeedle2 } \\
\text { positions in } \\
\text { 3D image }\end{array}$ & Otsu Ptrsu & $\begin{array}{l}\text { Tissue-equivalent } \\
\text { ultrasound prostate } \\
\text { phantom model } 053\end{array}$ & $\begin{array}{l}\text { CT/Fluoroscopic } \\
\text { scan images }\end{array}$ \\
\hline Holupka et al. ${ }^{\text {RNN }}$ & $\begin{array}{l}\text { Loose seeds } \\
\text { positions in } \\
2 \mathrm{D} \text { image }\end{array}$ & Deep learning & Clinical & CT images \\
\hline Golshan et al. pels & $\begin{array}{l}\text { Stranded seeds } \\
\text { han2020automatic } \\
\text { positions in } \\
\text { 3D image }\end{array}$ & Deep learning & Clinical & US images \\
\hline Dehghan et al. EMe & $\begin{array}{l}\text { Loose seeds } \\
\text { nhanced } \\
\text { positions in } \\
\text { 3D image }\end{array}$ & $\begin{array}{l}\text { US seed detection } \\
\text { enhanced by } \\
\text { EM-seed track }\end{array}$ & Yezitronix phantom & CT images \\
\hline \multirow{2}{*}{ This study } & \multirow{2}{*}{$\begin{array}{l}\text { Loose and } \\
\text { stranded seeds } \\
\text { positions }+ \text { orientations } \\
\text { in } 3 \mathrm{D} \text { images }\end{array}$} & \multirow{2}{*}{$\begin{array}{l}\text { Machine learning } \\
\text { and rigid registrations } \\
\text { methods }\end{array}$} & Agar-agar phantom & \multirow{2}{*}{$\begin{array}{l}\text { Manual } \\
\text { localization } \\
\text { of the US } \\
\text { images }\end{array}$} \\
\hline & & & Clinical & \\
\hline
\end{tabular}


Table 2: Compositions of stranded seeds released by the tranded_seeds_compositions needles in the phantom.

\begin{tabular}{|l|l|}
\hline Needle & Released stranded seeds composition \\
\hline N1 & Seed + Spacer + Seed \\
\hline N2 & $2 \times$ Seed + Spacer + Seed \\
\hline N3 & Seed $+2 \times$ Spacer + Seed \\
\hline N4 & $2 \times$ Seed + Spacer $)+$ Seed \\
\hline N5 & $3 \times$ Seed \\
\hline N6 & $2 \times$ Seed + Spacer + Seed $+2 \times$ Spacer + Seed \\
\hline N7 & $2 \times$ Seed + Spacer $+2 \times$ Seed \\
\hline N8 & Seed \\
\hline
\end{tabular}

Table 3: Statistics of localization error on 3D US images of two patients and error of estimated orientations of seeds tab_Statistics composing the strands $\mathrm{Si} ; \mathrm{i} \in[1 ; 4]$.

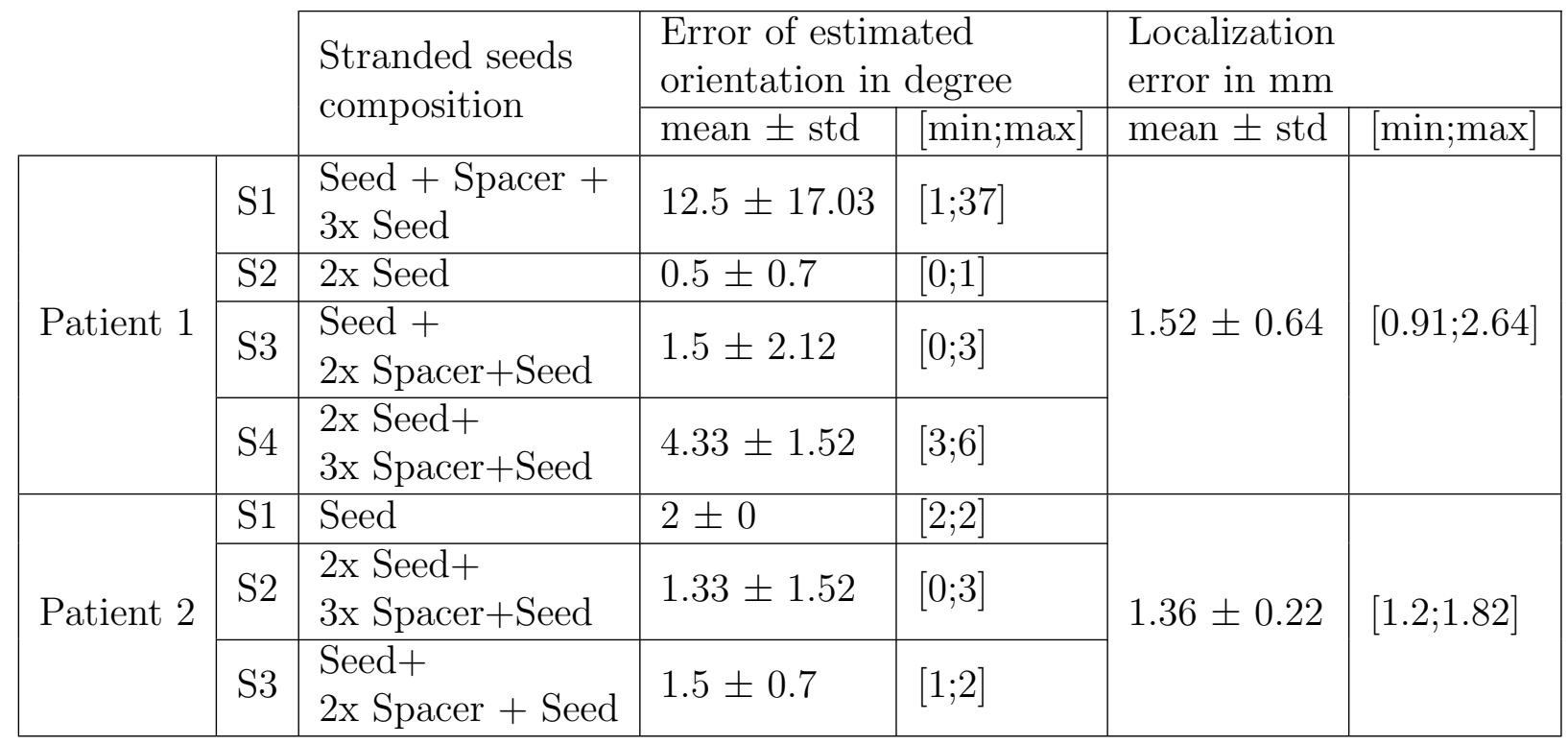


Table 4: Parameters values used for each image type acquired by two different US probes. The EM and SVM tab:parameters parameters are unitless.

\begin{tabular}{|l|l|l|l|l|l|l|l|l|l|}
\cline { 2 - 9 } \multicolumn{1}{c|}{} & \multicolumn{4}{l|}{ EM Initialization } & \multicolumn{2}{l|}{ SVM } & ICP \\
\cline { 2 - 10 } \multicolumn{1}{c|}{} & $\mu_{X_{T G}}$ & $\mu_{X_{B}}$ & $\sigma_{X_{T G}}$ & $\sigma_{X_{B}}$ & $\pi_{X_{T G}}$ & $\pi_{X_{B}}$ & $C$ & tol & threshold $\epsilon_{I C P}$ \\
\hline Phantom image & 235 & 100 & 3 & 40 & 0.05 & 0.95 & 0.15 & $10 \mathrm{e}-3$ & $0.01 \mathrm{~mm}$ \\
\hline Clinical images & 255 & 80 & 8 & 40 & 0.2 & 0.8 & 0.15 & $10 \mathrm{e}-3$ & $0.01 \mathrm{~mm}$ \\
\hline
\end{tabular}




\section{${ }_{596}$ VII. Figures}

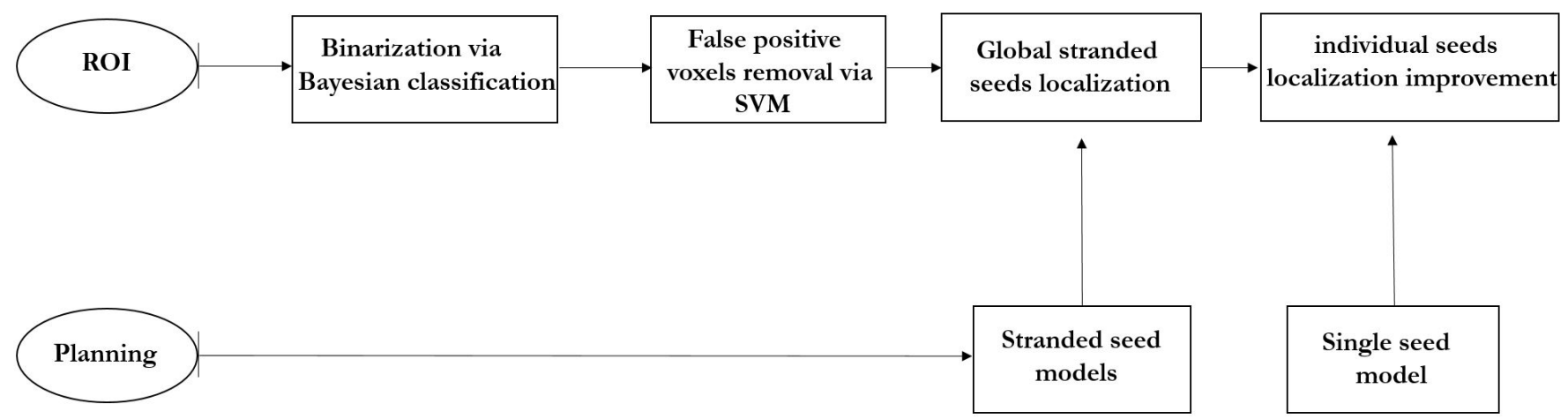

Figure 1: Workflow for oriented seeds localization. 


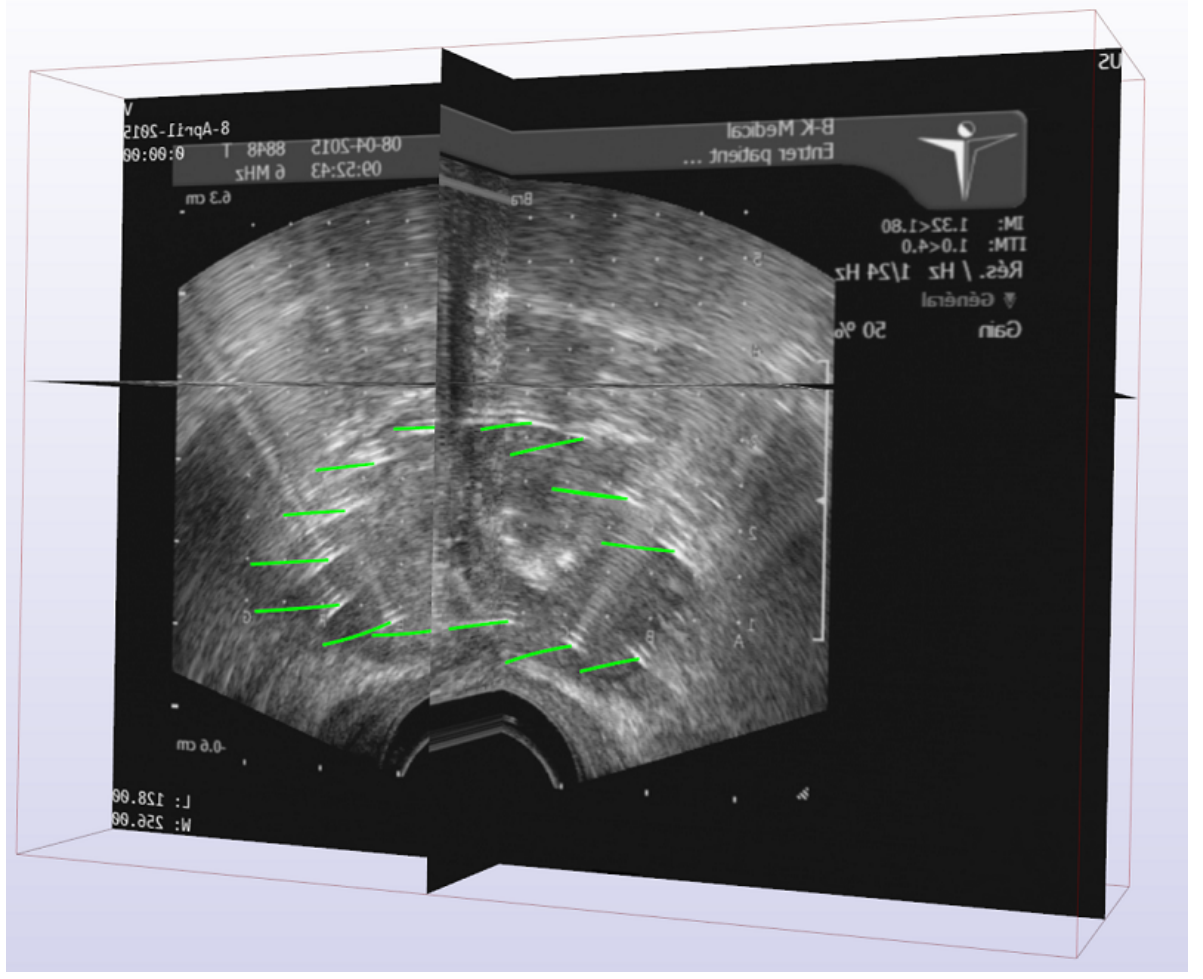

Figure 2: Detection of needles (green curves) in clinical images using needle detection method 


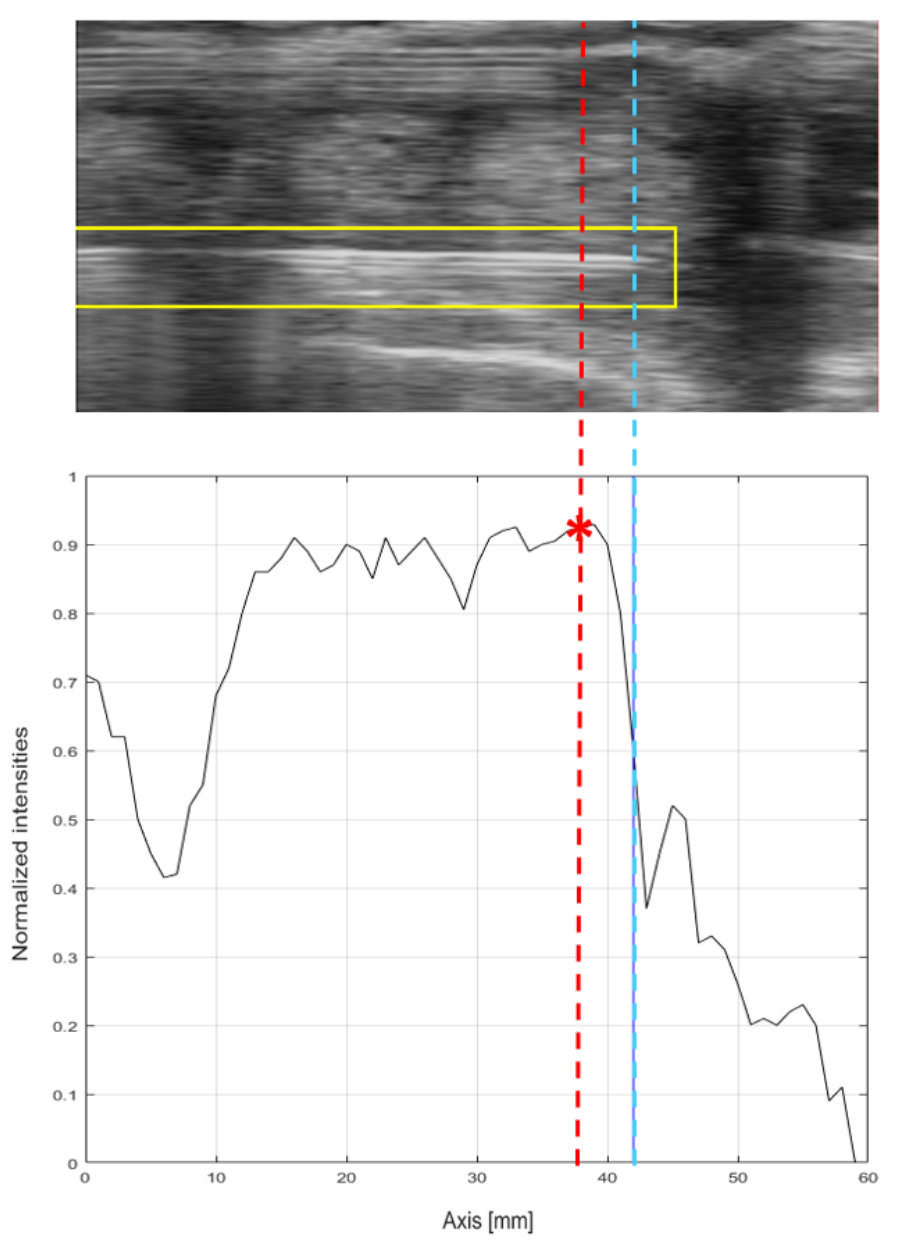

Figure 3: Needle tip localization example. Successful automatic localization despite a shadow region [3mm-15mm] where the needle's voxels intensities decrease significantly. The blue line corresponds to the first pixel of the needle shaft belonging to the background class (Bayes rule of inequality 2). The end of the needle initially detected is presented by the broken line passing through the red star. 


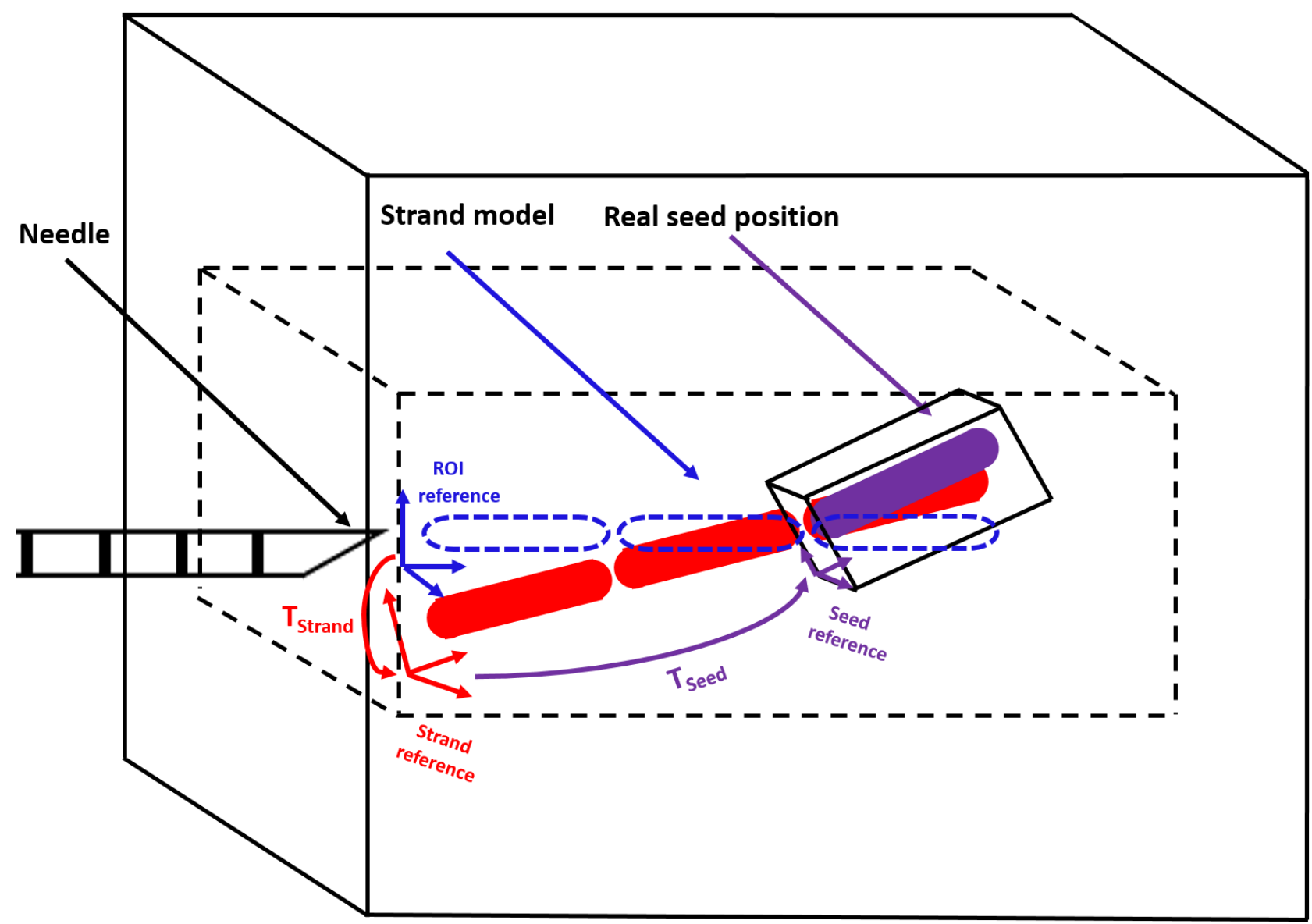

Figure 4: Reference frames and rigid transforms used by the method: global image, ROI, strand and seed references frames - positions of the strand and the individual seeds are encoded using respectively $T_{\text {Strand }}$ and $T_{\text {Seed }}$. 


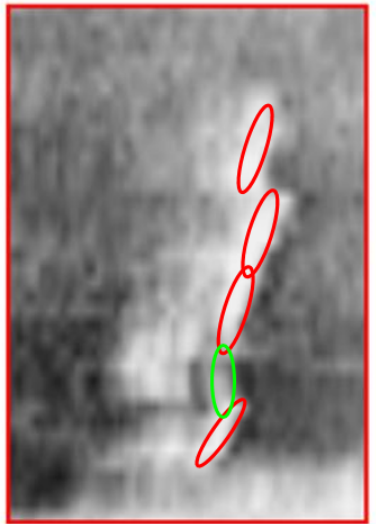

(a)

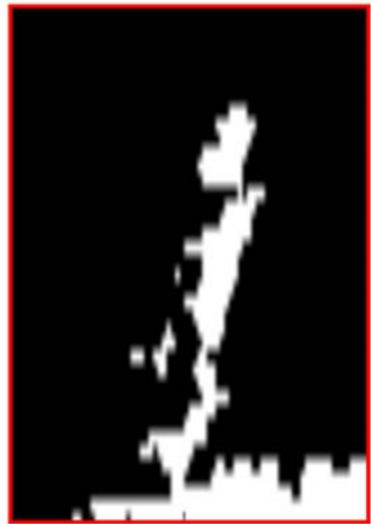

(b)

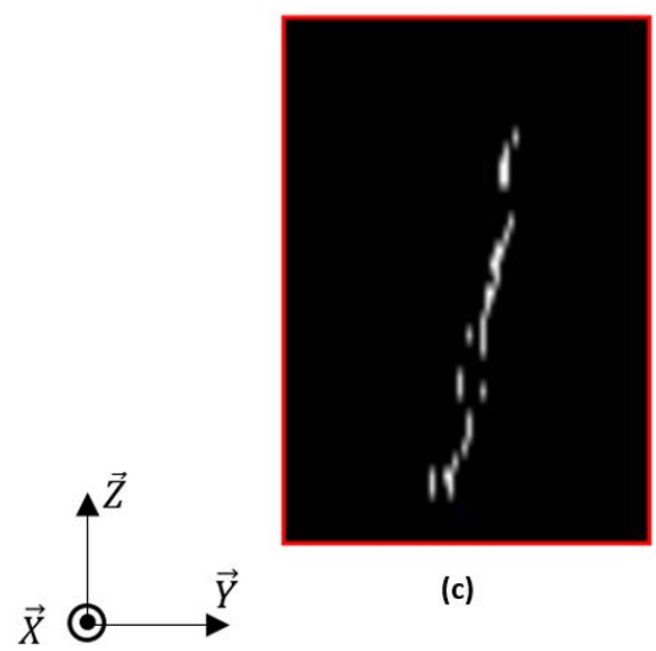

Figure 5: Sagittal sections of 3D clinical images containing stranded seeds. (a) manual segmentation based on planning information (seeds in red and spacer in green) added to the original image, (b) image after binarization with Bayesian classifier, (c) image after false positive removal with SVM classification. 


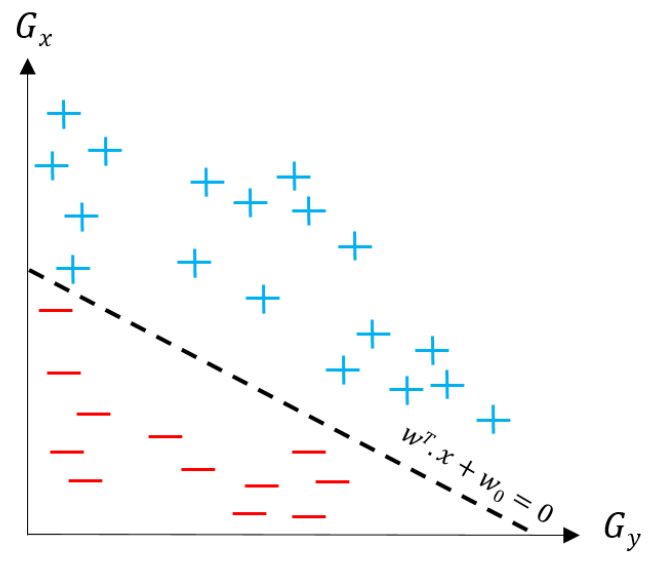

Figure 6: Example of SVM binary classification. True positives in blue fig:hyperplane and false positives in red.
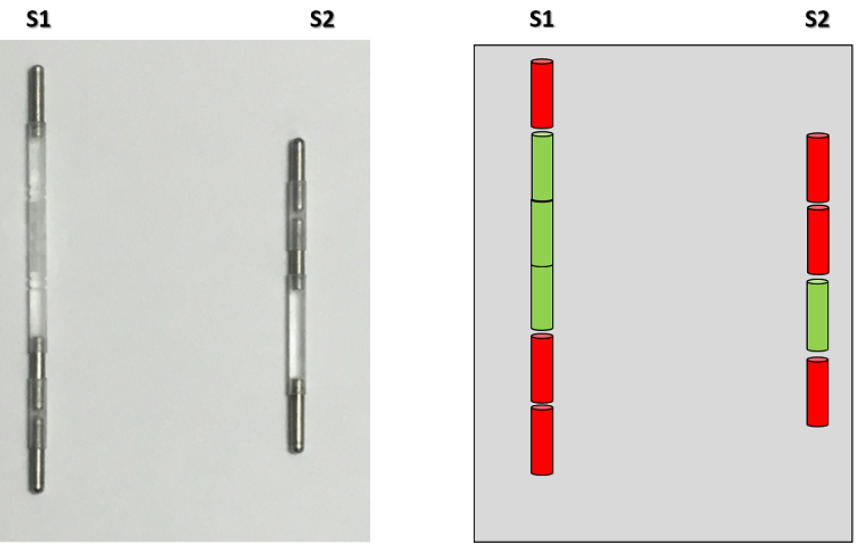

Figure 7: Two stranded seeds S1,S2 (left) with their model (right). Red cylinders represent radioactive seeds and green ones are for spacers and linkers. 


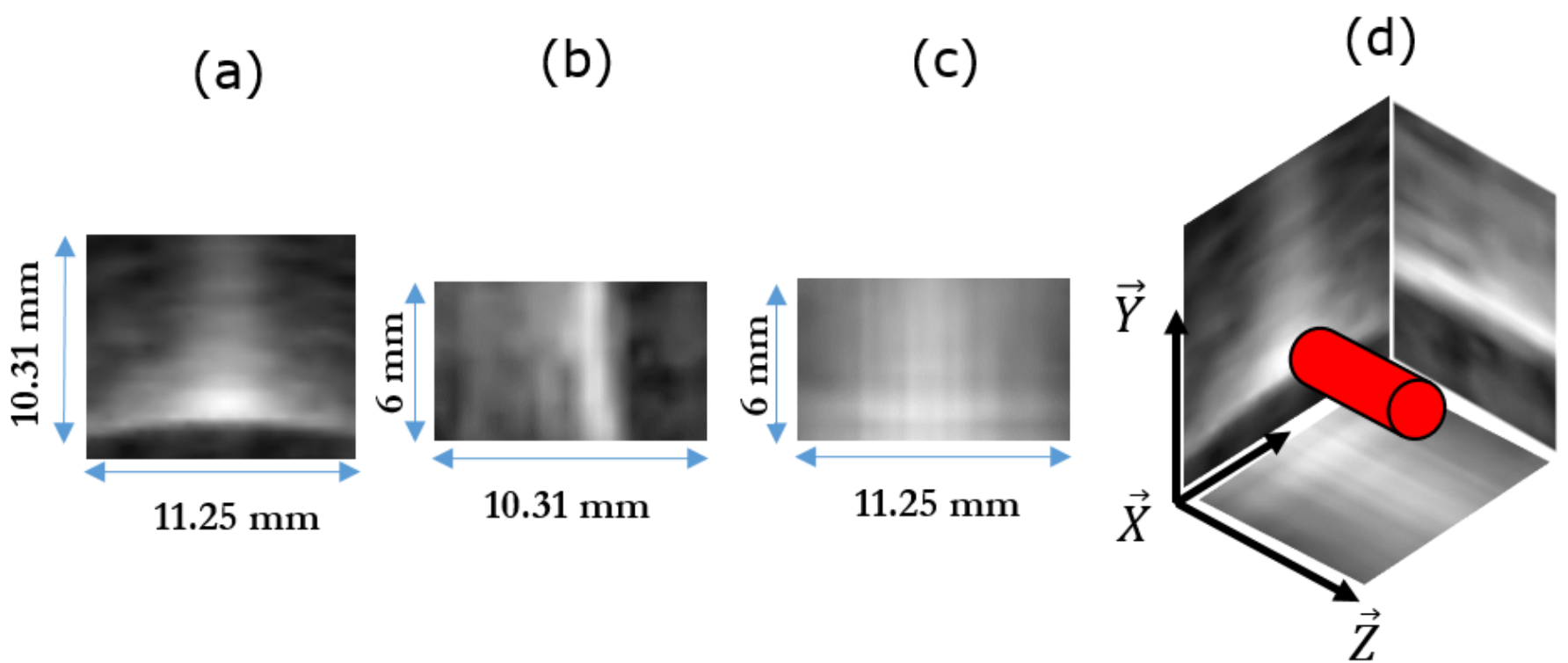

Figure 8: Artifacts in the 3 planes containing the seed in $V_{m}$ of phantom image. (a) axial plane, (b) sagittal plane, (c) transversal plane. $\mathrm{fig}$ :VS The seed visualized in $(\mathrm{d})$ is perpendicular to the axial plane.

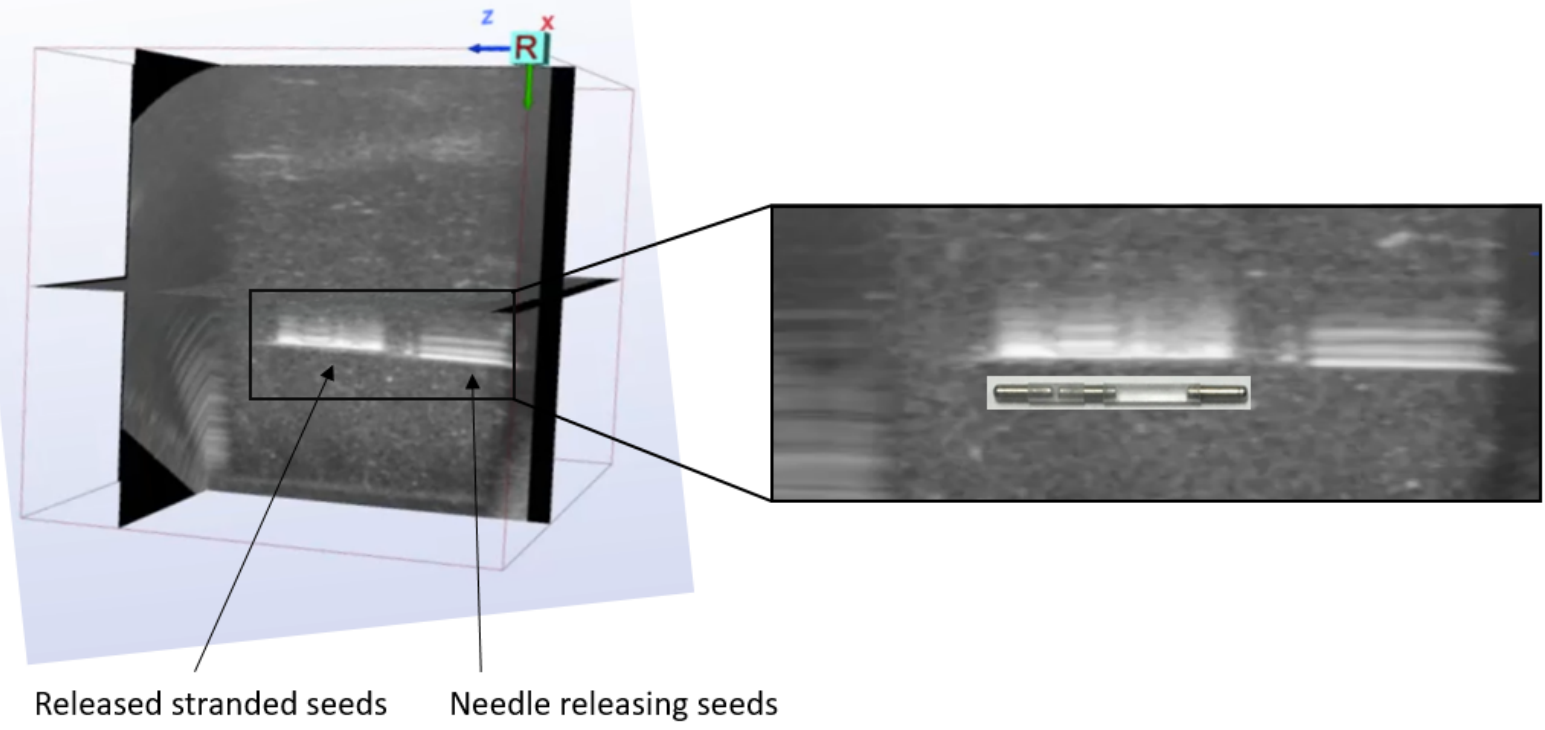

Figure 9: 3D US phantom image showing a needle during releasing stranded seeds. 

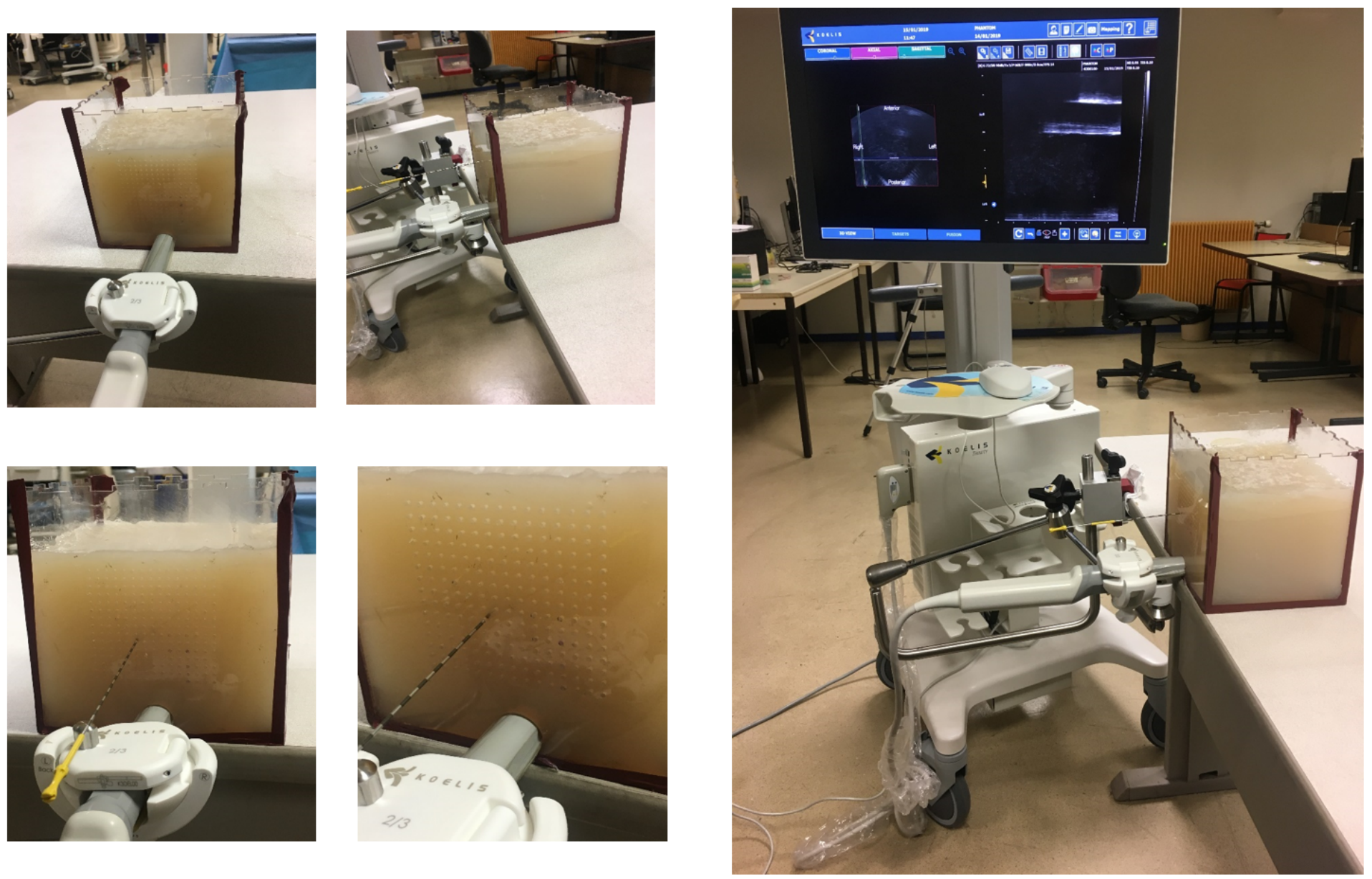

Figure 10: Experiments on Agar-agar phantom contained in a plastic box using the ultrasound probe of the TRINITY ${ }^{\circledR}$ (Koelis, Meylan, France) assistance workstation. 

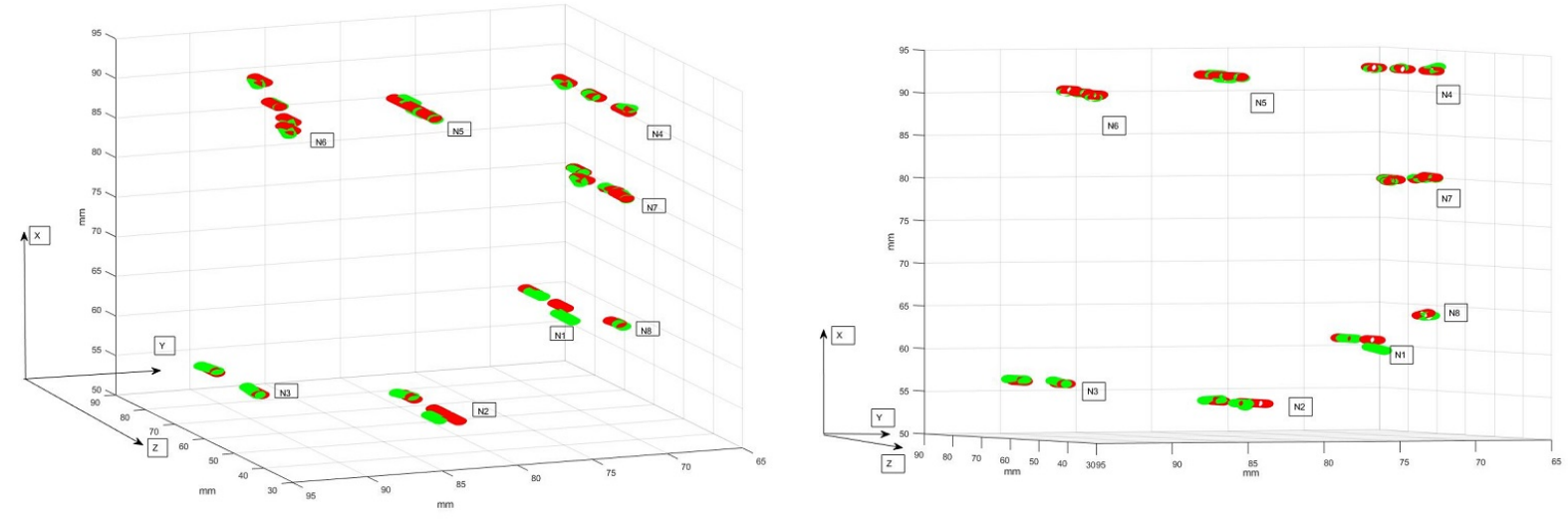

Figure 11: Seed localization result compared with manual identification ground truth at two different viewpoints. The red cylinders correspond to automatic seeds localization and the green cylinders to the ground truth. Names of the strands ( $c f$ table 2) are next to them. 


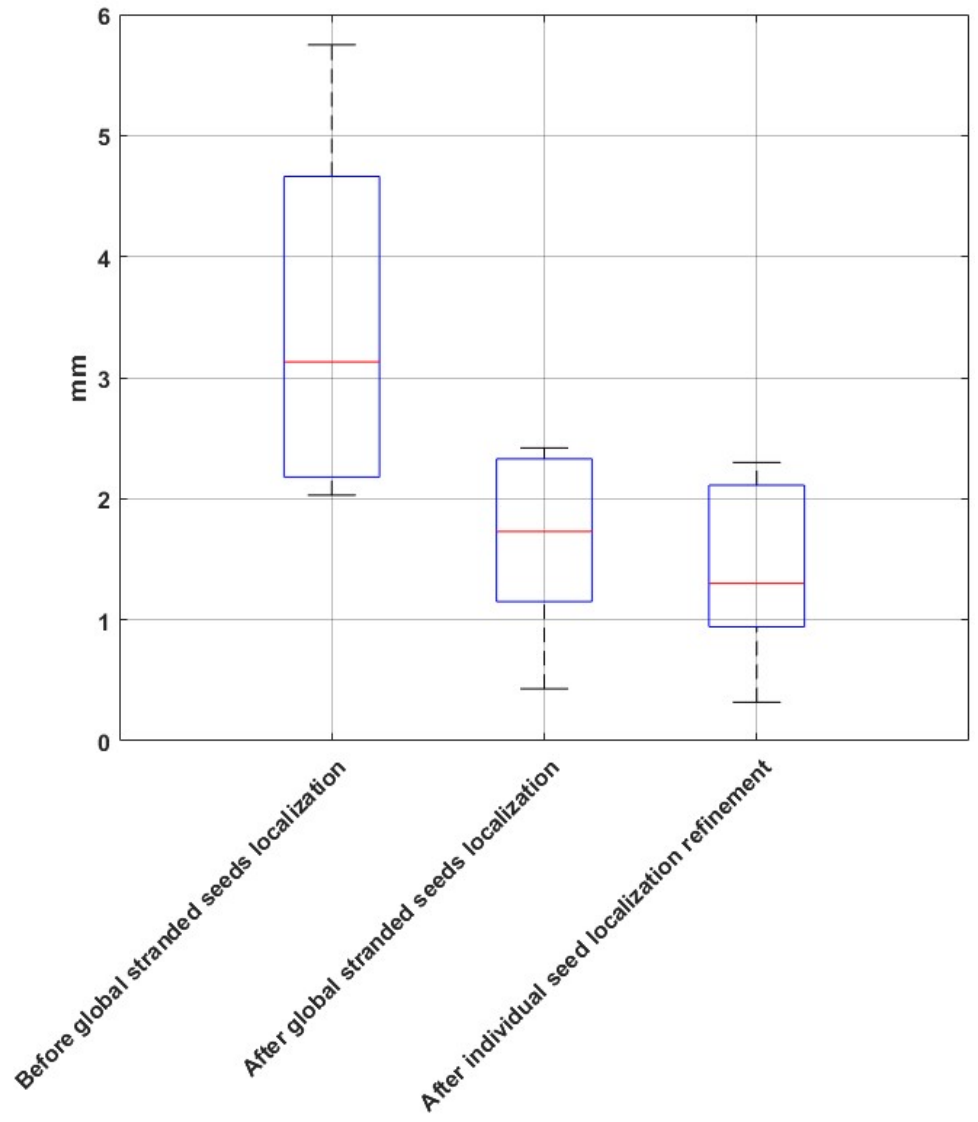

Figure 12: Boxplots presenting the distributions of the seeds localization errors in phantom images at the different stages of the method. The boxplots visualize the minimum and maximum scores (blue lower and upper dashes), the first and third quartiles (blue boxes) and median fig:boxplotPhantom (red dash). 


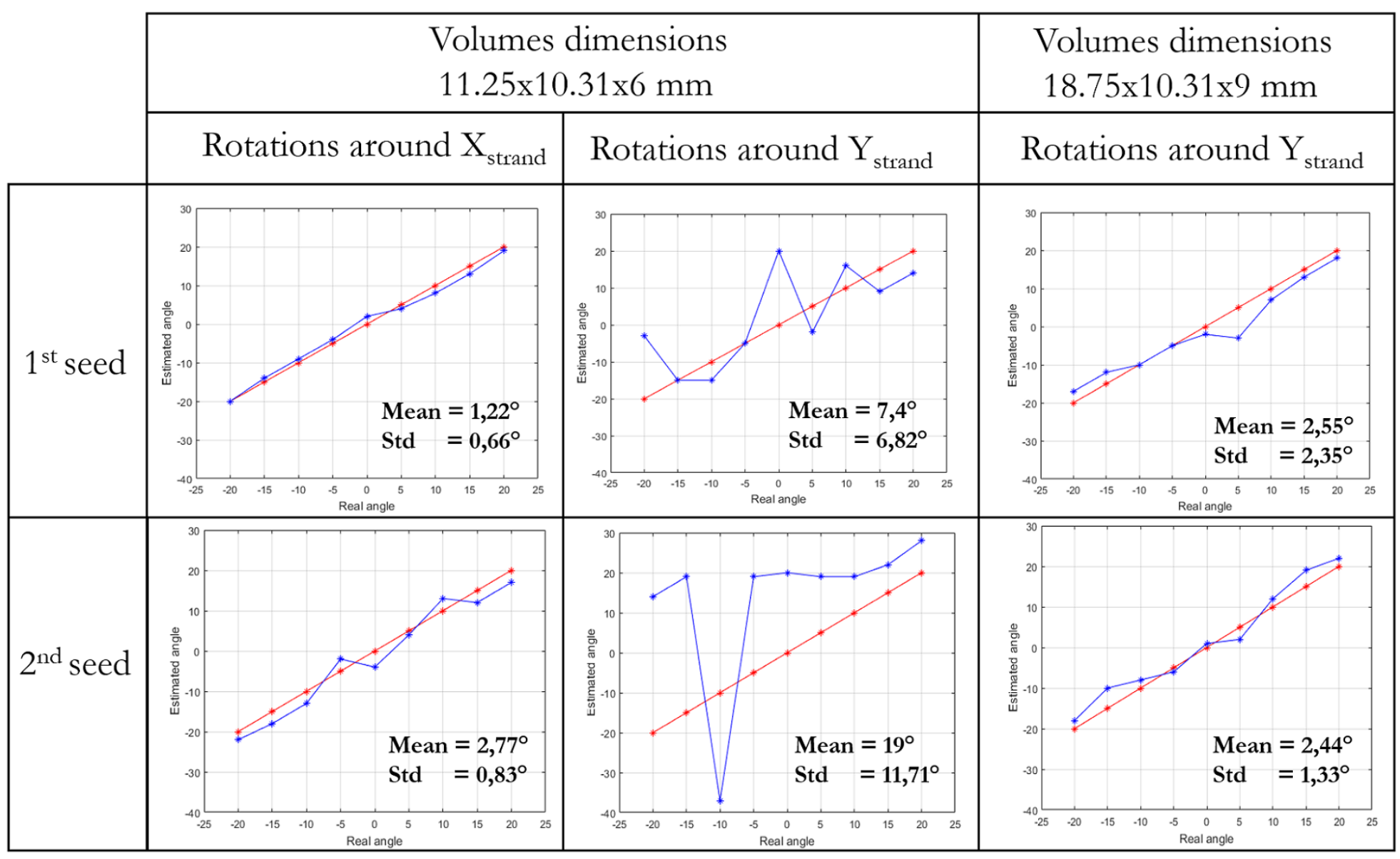

Figure 13: Estimation of the orientations of two seeds with different volumes dimensions. \{Red,Blue\} points are respectively the fig:orientations \{applied,estimated $\}$ angles. 


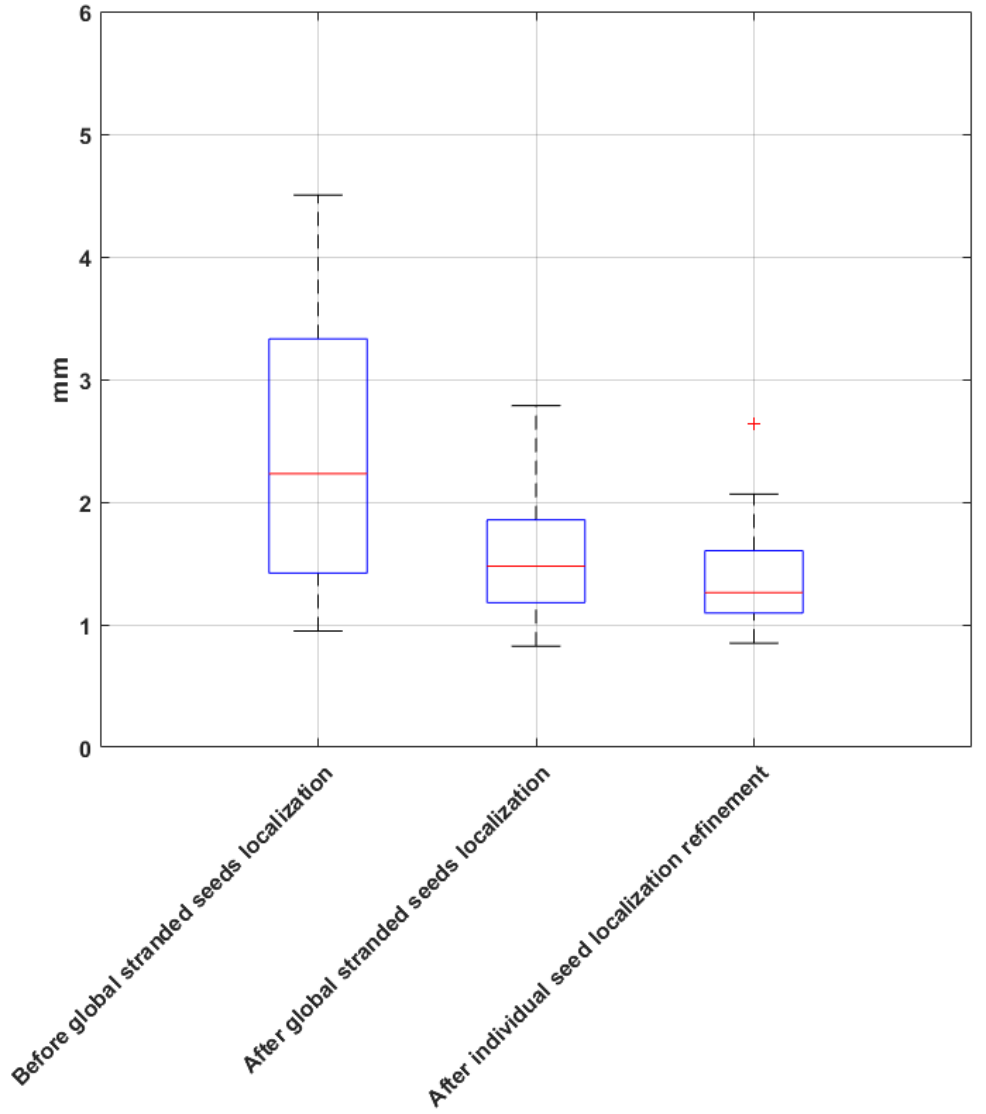

Figure 14: Boxplots presenting the distributions of the seeds localization errors in clinical Lotpatients images at the different stages of the method. 